\title{
Skin nerve sympathetic activity during insulin-induced hypoglycaemia
}

\author{
C. Berne ${ }^{1}$ and J. Fagius ${ }^{2}$ \\ Departments of Internal Medicine ${ }^{1}$, Neurology $y^{2}$ and Clinical Neurophysiology ${ }^{2}$, Uppsala University, Akademiska Sjukhuset, Uppsala, Sweden
}

\begin{abstract}
Summary. Microelectrode recordings of skin nerve sympathetic activity, consisting of sudomotor and vasoconstrictor signals, were performed in the peroneal nerve in seven healthy subjects during insulin-induced hypoglycaemia. The nerve activity was recorded at rest and for $90 \mathrm{~min}$ after intravenous injection of $0.15 \mathrm{IU}$ insulin $/ \mathrm{kg}$ body weight. The net outflow of skin nerve sympathetic activity was increased during hypoglycaemia, with the exception of one subject who exhibited a high initial level of activity. In all subjects a change of the temporal pattern of the outflow was found, suggesting a shift from mixed (sudomotor and vasoconstrictor) to pure sudomotor activity. This change coincided with a sensation of warmth,
\end{abstract}

sweating and varying degrees of cutaneous vasodilatation, and was followed by a fall in body temperature. It is concluded that hypoglycaemia has a differential effect on sympathetic activity in skin nerves, with a strong increase of sudomotor impulses and simultaneous inhibition of vasoconstrictor signals. Thus, neurally mediated thermoregulatory adjustment contributes to heat loss during hypoglycaemia.

Key words: Hypoglycaemia, sympathetic nervous system, skin nerve sympathetic activity, microelectrode recording, thermoregulation.
During insulin-induced hypoglycaemia, sweating, changes of cutaneous blood flow and reduction of body temperature occur, strongly suggesting activation of sympathetic mechanisms for thermoregulation. However, the precise mode of sympathetic nerve discharge to skin effector organs during hypoglycaemia is unknown.

Microelectrode recording provides direct access to sympathetic nerves in the limbs in alert, cooperating subjects [1]. Sympathetic nerve activity has been shown to occur as multiunit volleys of impulses with interposed intervals of neural silence, displaying different characteristics in muscle and skin nerve fascicles. Muscle nerve sympathetic activity (MSA), which is involved in cardiovascular regulation, was recently shown to increase considerably during insulin-induced hypoglycaemia [2]. Skin nerve sympathetic activity ( $S S A)$ consists of sudomotor and vasoconstrictor impulses. It is of importance for thermoregulation and is also activated by arousal stimuli and emotional stress [3, 4]. SSA manifests itself as irregular bursts of impulses generated in an unpredictable fashion, unlike MSA, which is strictly coupled to the cardiac rhythm. At rest and in response to arousal stimuli, SSA appears in parallel in arms and legs in nerves subserving similar cutaneous regions [5].

The present study was undertaken to characterize SSA during insulin-induced hypoglycaemia.

\section{Subjects and methods}

\section{Subjects}

Skin nerve sympathetic activity was recorded in seven healthy volunteers, five men and two women, aged 23-41 (mean 29) years. The study was approved by the Ethics Committee of the Medical Faculty, University of Uppsala, and informed consent was obtained from all subjects.

\section{Nerve recordings}

An insulated tungsten microelectrode (tip diameter about $5 \mu \mathrm{m}$ ) was inserted manually through the intact skin into the underlying right peroneal nerve at the fibular head. A low impedance reference electrode was placed subcutaneously at a distance of $1-2 \mathrm{~cm}$. The nerve was localized by electrical stimuli delivered through the recording electrode. When the nerve was encountered, the electrode was positioned within a skin nerve fascicle. This position was found by the subject reporting paraesthesia without concomitant muscle twitching being evoked by the electrical stimuli, and by the appearance of afferent, mechanoreceptor activity elicited by gently touching the skin innervated by the nerve. Thereafter minor adjustments of the electrode position were made until the characteristic pattern of multi-unit SSA (see Figs. $2 \mathrm{~A}$ and $3 \mathrm{~A}$ ) was recorded. The evidence for a sympathetic origin of the recorded signals has been previously summarized [1].

The nerve recording procedure may cause minor discomfort during the search for the nerve. Once a suitable electrode position is found, no discomfort is experienced during the recording. The recordings caused no undesirable effects.

The nerve signal was amplified in two steps (total gain 50,000 $\times$ ) and fed through a $700-2000 \mathrm{~Hz}$ band pass filter and an amplitude dis- 
criminator for improving signal-to-noise ratio. An RC integrating network with time constant $0.1 \mathrm{~s}$ delivered a mean voltage display of the multi-unit neural activity.

$E C G$ was recorded by chest electrodes and respiratory movements by a strain gauge strapped around the chest with a rubber band. A photoelectric plethysmograph (van Gogh, Amsterdam, Netherlands) was used for monitoring skin blood flow changes in the big toe of the right foot.

All recorded signals were stored on tape (FM tape recorder Sangamo Sabre VI, Sangamo Weston-Schlumberger, Sarasota, Fla, USA) for subsequent analysis. During the experiments the signals were displayed on a storage oscilloscope.

\section{Blood chemistry}

Blood glucose was measured with glucose dehydrogenase (E. Merck AG, Darmstadt, FRG) [6] in an automated analysis system (Greiner G-300, Langenthal, Switzerland). The catecholamine concentration in venous plasma was determined by high performance liquid chromatography using a cation exchange column and electrochemical detection [7].

Blood samples for catecholamine analysis were collected in $10 \mathrm{ml}$ ice-chilled vacutainer tubes to which $0.2 \mathrm{ml}$ of glutathione and EGTA had been added to yield a final concentration of $4 \mathrm{mmol} / 1$ and $5 \mathrm{mmol} / 1$, respectively (chemicals from Sigma Chemical Company, $\mathrm{St}$ Louis, Mo, USA). After centrifugation at $+4{ }^{\circ} \mathrm{C}$ serum was immediately frozen and kept at $-20^{\circ} \mathrm{C}$ until analysis.

\section{General procedure}

The experiments were carried out in a laboratory with an ambient temperature of $22-24^{\circ} \mathrm{C}$. Subjects were fasting and did not smoke after midnight. The experiments were started at 08.00 hours with the subjects lying supine on a comfortable bed. After insertion of separate indwelling intravenous catheters for blood specimens and insulin injection, the search for a suitable intraneural electrode position lasted 10-60 min. After recording SSA at rest for 15-30 min, hypoglycaemia was induced by rapid intravenous injection of regular insulin (Actrapid, NOVO A/S, Bagsvaerd, Denmark), $0.15 \mathrm{IU} / \mathrm{kg}$ body wt., and the sympathetic signals were then recorded continuously throughout the experimental procedure. Blood samples for the analyses of blood glucose and catecholamines were drawn at $-15,0,15,30,45,60,75$, and $90 \mathrm{~min}$ relative to the insulin injection. Thus, the whole experiment could last up to $3 \mathrm{~h}$, during which time the subjects were kept in the supine position.

In four subjects evaluation of central core temperature was made by insertion of a thermistor probe (Exacon System 9000 , Exacon Scientific Instruments APS, Taastrup, Denmark; accuracy $<0.1^{\circ} \mathrm{C}$ ) into the external acoustic meatus and in five subjects skin temperature of the big toe was measured with a similar thermistor probe.

Subjects were advised to relax and to report symptoms of hypoglycaemia.

\section{Analysis procedure}

A $2.5 \mathrm{~mm} / \mathrm{s}$ paper display of the recorded signals was produced by an ink-jet recorder (Siemens-Elema, Stockholm, Sweden). The mean voltage neurogram was amplified such that a similar amplitude of bursts in all recordings was achieved; i. e. amplification differed between subjects due to different electrode positions. Detailed analysis was restricted to $6 \mathrm{~min}$ periods, parallel with the drawing of blood samples. Heart rate was determined during the selected periods of analysis.

As the strength of a recorded burst (corresponding to the area under the burst in the mean voltage neurograms) is critically dependent on the intraneural position of the electrode, and as the bursts occur irregularly, there is no appropriate way to measure the amount of SSA. This precludes quantitative inter-individual comparison or compari- son between two recordings in one individual. Within a given recording, however, the relative area under the neurogram is representative for the strength of the activity, provided the electrode position remains unchanged $[8,9]$. Thus, the area under the neurogram to an added baseline was measured in the periods of analysis; a mean value per minute, expressed in arbitrary units, was obtained for each period (at $90 \mathrm{~min}$, the original electrode position was lost in two subjects). The area under the neurogram was measured using a digitizing board (Talos, Phoenix, Ariz, USA) connected to a computer (Apple Computer, Cupertino, Calif, USA). The noise level of the neurogram, corresponding to no detectable SSA, was found to be about 15 arbitrary units/min (Fig. 2B and 3B).

During initial rest and during hypoglycaemia the duration (s) of 200 consecutive bursts of SSA was measured in each subject by use of another digitizing board (Hipad, Houston Instruments, Austin, Tex, USA; computer Digital Dec 11/40, Digital Equipment, Maynard, Mass, USA).

The latter equipment was also used for measurement of relative pulse amplitude in the big toe. During each analysis period of about $6 \mathrm{~min}, 40$ pulse waves were measured and selected as every 10 th of 400 consecutive pulse waves, resulting in a mean pulse amplitude (arbitrary units) for each analysis period.

A computerized waveform analyser (Data 6000, Data Precision, Danvers, Mass, USA) was used for detection of SSA rhythms coupled to the cardiac rhythm. An averaging of 64 superposed 2-s segments of the mean voltage neurogram, triggered by the $R$ wave of the $E C G$, was performed.

Respiratory rates were obtained from the paper display during initial rest, at nadir of blood glucose and after counterregulation had occurred.

\section{Statistical analysis}

Results are expressed as mean \pm SEM. Student's t-test for paired and unpaired observations was employed for statistical assessment. A $p$ value of 0.05 was considered statistically significant.

\section{Results}

The time courses for blood glucose, net outflow of SSA, skin circulation in the toe and body temperature are summarized in Figure 1.

Mean fasting blood glucose before insulin injection was $4.6 \mathrm{mmol} / \mathrm{l}$ (range $3.8-5.4$ ). Blood glucose reached a nadir at $30 \mathrm{~min}(1.5 \mathrm{mmol} / 1$ with a range of $0.7-2.1)$. All subjects reported symptoms like drowsiness, feeling of hunger, warmth and palpitations during hypoglycaemia. The heart rate was increased 20 to $40 \mathrm{~min}$ after insulin injection with a short-lasting maximum at 25-30 min. Sweating, starting after $30 \mathrm{~min}$, was profuse in five and slight in two subjects.

Plasma noradrenaline and adrenaline increased as described for a comparable experimental protocol [2], confirming that the low blood glucose levels had activated counterregulatory mechanisms.

The level of SSA at rest varied among subjects (Figs. 2A, 3A). During maximal hypoglycaemia, the net outflow of SSA increased in most subjects (Fig. $2 \mathrm{~A}$ and $\mathrm{B})$, and was significantly higher at 30 and $45 \mathrm{~min}(\mathrm{p}<$ 0.05 ) compared with time 0 (Fig. 1). The response varied widely, however, and in one subject the net outflow of SSA did not increase at all, although the shape of the bursts changed considerably (Fig. $3 \mathrm{~B}$ ). 

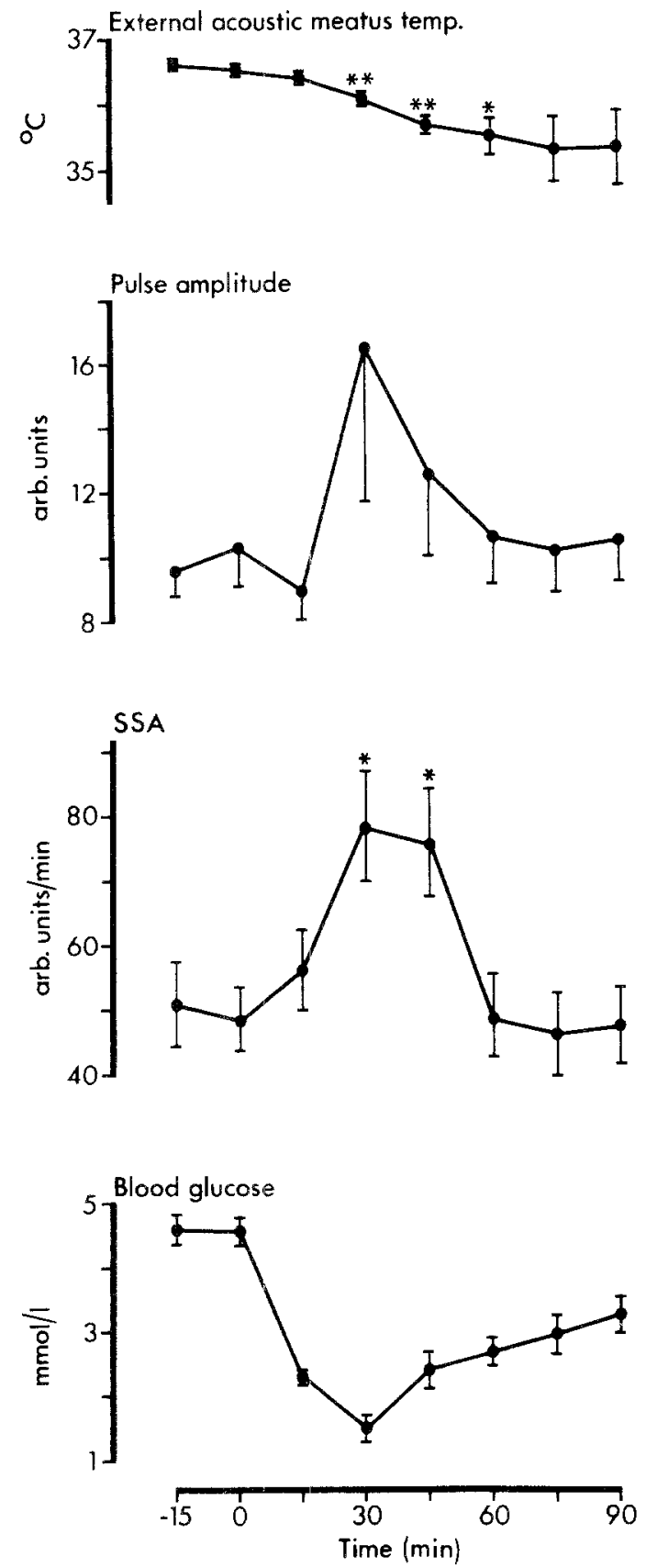

Fig. 1. Time course of insulin-induced changes of blood glucose (bottom; $n=7$ ), net outflow of skin nerve sympathetic activity (SSA) (second; $n=7$; at $90 \min n=5$ ), relative pulse amplitude in skin of the big toe (third; $n=7$ ) and external acoustic meatus temperature (top; $n=4$ ). Intravenous injection of insulin at time 0 . Significance levels (not indicated for blood glucose): ${ }^{*} p<0.05 ;{ }^{*} p<0.01$

The most prominent effect on SSA was a shortening of the duration of single bursts (see Fig. $2 \mathrm{~A}$ and $3 \mathrm{~A}$ and histograms in Fig. $2 \mathrm{C}$ and $3 \mathrm{C}$ ), occurring in all subjects, with a rather sudden onset at 25-32 min and lasting for 14-23 (mean 18) min. The change was highly significant in each subject $(p<0.001)$. This shift of temporal pattern coincided with the sensation of warmth and was followed by sweating.

ECG-triggered averaging of the mean voltage neurogram showed no or only a slight influence of the car- diac rhythmicity on nerve activity under normal conditions. During hypoglycaemia a pulse-synchronous rhythmic component was clearly detectable in all subjects but one.

All subjects but one exhibited some degree of vasodilatation in the big toe during hypoglycaemia (Fig. 1), coinciding with the increase and change of temporal pattern of SSA. In some subjects the dilatation was large (Fig. 3A). Similar observations were made in another series of seven subjects, in which MSA was recorded during hypoglycaemia [2]. When the blood glucose returned towards normal level the pulse amplitude tended to assume its initial size.

External acoustic meatus temperature $(n=4)$ fell during hypoglycaemia (Fig.1) and in three subjects it showed a tendency to increase after $75 \mathrm{~min}$. Skin temperature of the big toe exhibited small and bidirectional changes, except in the subject with extreme vasodilatation, whose temperature rose by $5.5^{\circ} \mathrm{C}$ during hypoglycaemia. In the remaining subjects no correlation was observed between vasodilatation and skin temperature.

Respiratory rate increased during hypoglycaemia (Fig. $2 \mathrm{~A}$ and $3 \mathrm{~A}$ ), from $14.2 \pm 1.6$ to $21.7 \pm 1.7$ breathing cycles $/ \min (p<0.001)$, and returned to the initial rate when counterregulation occurred. The depth of breathing was essentially unchanged during the procedure with the exception of a few instances of brief hyperventilation (excluded from analysis) when hypoglycaemic symptoms first appeared.

\section{Discussion}

A main finding in the present study was an increment in the net outflow of skin nerve sympathetic activity elicited by insulin-induced hypoglycaemia (Figs. 1 and 2). One subject, who did not show this response, already had a high level of SSA while at rest (Fig. 3). This was probably due to tension associated with the experimental situation; tension is known to stimulate SSA [4]. Therefore, it seems safe to conclude that the net outflow of SSA is enhanced by insulin-induced hypoglycaemia provided that true resting conditions are obtained at the outset of the experiments.

A conspicuous effect, observed in all subjects, was a change of the temporal pattern of SSA from broad to narrow bursts (Figs. 2 and 3). Previous studies [10,11] have shown that sudomotor impulses are conducted with a higher velocity than vasoconstrictor impulses. This results in a smaller temporal dispersion of pure sudomotor bursts than of mixed or vasoconstrictor bursts. Thus, the occurrence of only narrow bursts during hypoglycaemia strongly suggests that the activation of SSA was restricted to sudomotor impulses, whereas vasoconstrictor activity was inhibited. The simultaneous profuse sweating and cutaneous vasodilatation support this interpretation. 

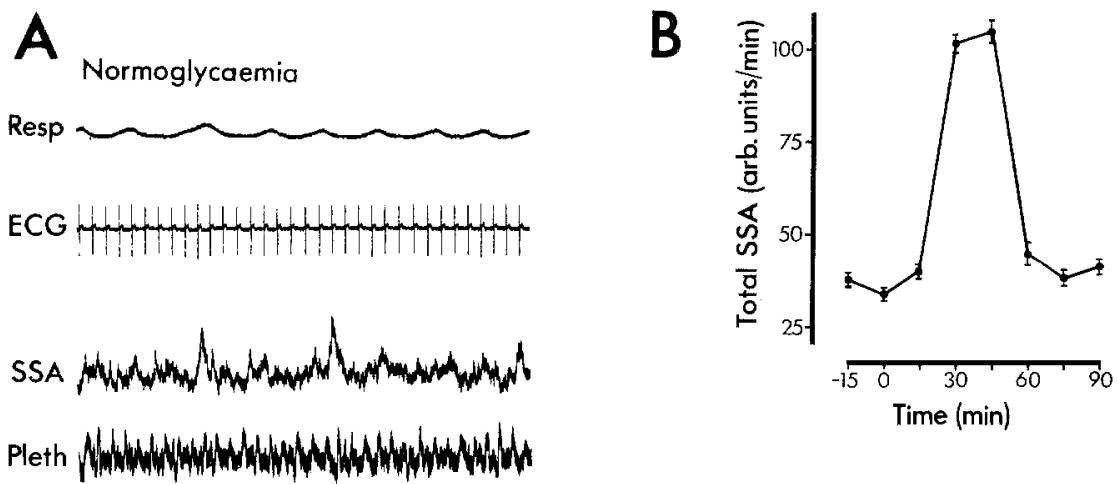

Hypoglycaemia

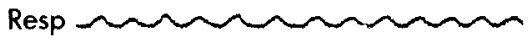
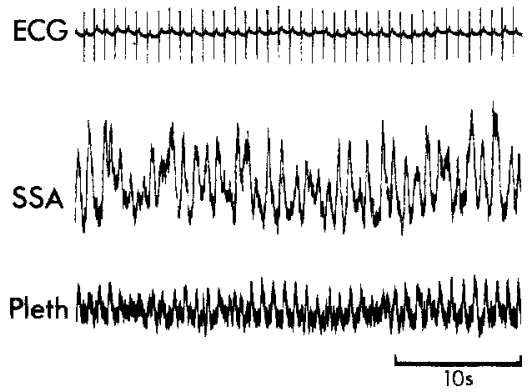

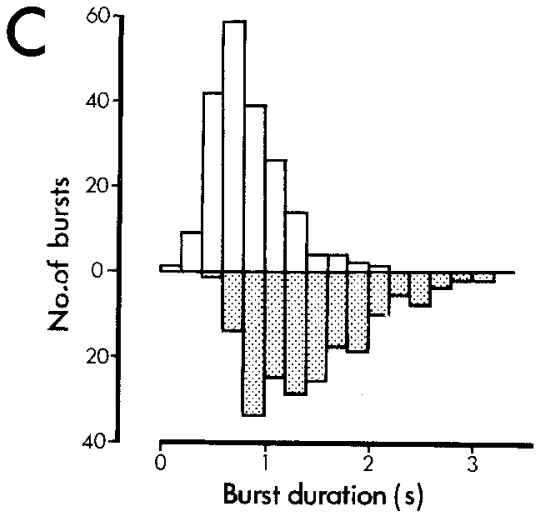

Fig. 2 A-C. SSA during insulin-induced hypoglycaemia in one subject. A Record examples before and following insulininduced hypoglycaemia. Resp - respiration. SSA - skin nerve sympathetic activity (mean voltage neurogram). Pleth - plethysmographic recording of pulse amplitude in skin of the big toe. B Net outflow of SSA; intravenous injection of insulin at time 0 . C Distribution of burst durations during normoglycaemia (lower histogram) and hypoglycaemia (upper histogram). Each histogram based on 200 observations
Overt cardiac rhythmicity, a characteristic feature of muscle nerve sympathetic activity (MSA), is not seen in SSA [1]. However, cardiac rhythm was also found in SSA after strong stimulation of sudomotor activity by rising ambient temperature and using the same ECGtriggered averaging technique as employed in the present study [12]. Thus, the detection of a rhythmic component of SSA, time-locked in the cardiac cycle, during hypoglycaemia further supports that the sympathetic outflow mainly consisted of sudomotor impulses.

Only the peroneal nerve was explored in the present investigation. Since SSA appears in parallel in different extremity nerves subserving similar cutaneous regions [5], we assume that our findings in one nerve are representative for nerves subserving hands and feet. The effects observed in the present study did not appear until hypoglycaemia was pronounced, indicating that the changes of SSA were related to reduced blood glucose and not to insulin per se.

Hyperventilation evokes strong mixed (sudomotor and vasoconstrictor) bursts of SSA [4]. The present increase of respiratory activity was too small to account for the observed effects. Furthermore, as already discussed, the increase of SSA is probably due to sudomotor activation only.

Low body temperature is a well-known feature of hypoglycaemia [13, 14]. The present findings suggest that this is, at least in part, a consequence of activation of neuroregulatory mechanisms for cooling of the body. A possible explanation for these events might be an disproportional response of central thermoregulatory neu- rons to the increased thermogenesis occurring in hypoglycaemia [15]. Whether the increased thermogenesis in its turn is directly related to hypoglycaemia or to high levels of catecholamines [16] is unknown.

The observed inhibition of the vasoconstrictor component of SSA in hypoglycaemia, suggested previously [17], would imply an increase of skin blood flow. Such an increase did occur, but not always; it also varied widely between subjects, an observation consistent with previous studies $[14,18]$. Circulating adrenaline, enhanced in hypoglycaemia, exerts a constrictor effect on skin vessels [19]. The varying effect on skin circulation in hypoglycaemia thus may be ascribed to variations in the balance between vasodilatation due to withdrawal of sympathetic vasoconstrictor command and vasoconstriction caused by adrenaline. This notion is supported by the finding of exaggerated cutaneous vasoconstriction during hypoglycaemia in sympathectomized subjects, in whom there is no sympathetic vasoconstrictor output that can be inhibited $[17,20]$, and by increased hand blood flow during hypoglycaemia in adrenalectomized subjects in whom no adrenaline-induced vasoconstriction can occur [21].

Muscle nerve sympathetic activity (MSA), consisting of vasoconstrictor impulses [1], was recently shown to increase in both normal and adrenalectomized subjects during insulin-induced hypoglycaemia [2]. Thus, opposite responses occur in hypoglycaemia, namely an increase of vasoconstrictor impulses to muscle vessels and a simultaneous withdrawal of vasoconstrictor signals to skin vessels. These events are accompanied by 
A

Normoglycaemio

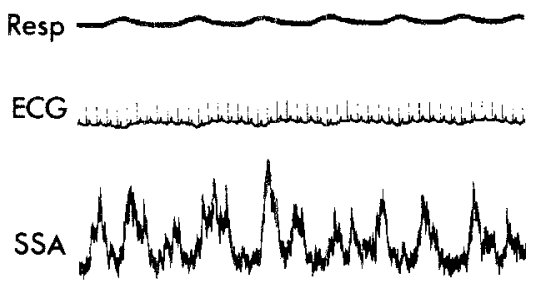

Pleth

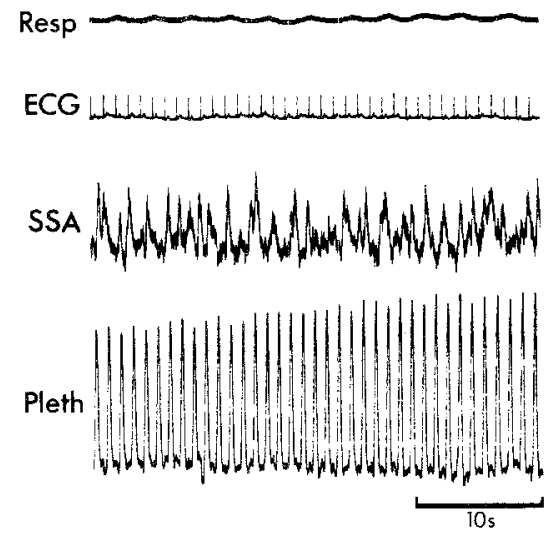

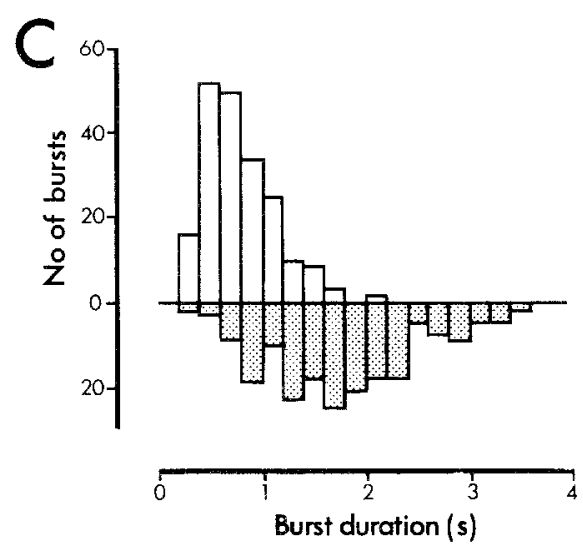

B

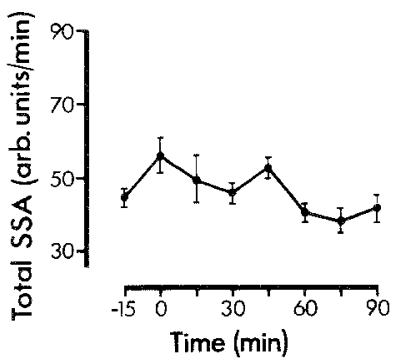

Fig. 3A-C. SSA during insulin-induced hypoglycaemia in one subject. See Figure 2 for explanations dilatation in muscle vasculature [21, 22], i.e. a seemingly contradictory phenomenon, whereas the tendency to skin vasodilatation is more logical. The dissociated response of the sympathetic nervous system to hypoglycaemia is further emphasized by the strong increase of the sudomotor component of SSA.

Such variation between different subdivisions of the sympathetic nervous system may explain the difficulties in detecting sympathetic activation in hypoglycaemia in adrenalectomized subjects by determination of noradrenaline levels [23, 24]. In such studies, increased noradrenaline release from one source and decreased release from another (the cholinergic, sudomotor component of SSA does not contribute to noradrenaline release), may result in an insignificant net change of noradrenaline levels.

The damage to the autonomic nervous system that occurs in longstanding diabetes [25] results in absent sympathetic activity in skin and muscle nerves in many cases [26]. This implies that in hypoglycaemia no activation can occur. An impaired sympathetic response to hypoglycaemia presumably contributes to the insufficient awareness of hypoglycaemia, which complicates treatment of diabetic patients with autonomic neuropathy. Whether a lack of response of the sympathetic nervous system is also involved in the defective counterregulation of hypoglycaemia in diabetes [27] remains to be determined.
Acknowledgements. Supported by the Swedish Medical Research Council, grant no. B86-04X-07468-01A, the Swedish Society of Medical Sciences, the Astrid Karlsson Fund for Medical Research, the Einar and Eva Lindberg Fund and the Ernfors Fund for Diabetes Research, and by grants from Novo Industries $A B$, Sweden, and the Swedish Diabetes Association.

\section{References}

1. Wallin BG, Fagius J (1986) The sympathetic nervous system in man - aspects derived from microelectrode recordings. Trends Neurosci 9:63-67

2. Fagius J, Niklasson F, Berne C (1986) Sympathetic outflow in human muscle nerves increases during hypoglycemia. Diabetes 35 : $1124-1129$

3. Hagbarth K-E, Hallin RG, Hongell A, Torebjörk HE, Wallin BG (1972) General characteristics of sympathetic activity in human skin nerves. Acta Physiol Scand 84: 164-176

4. Delius W, Hagbarth K-E, Hongell A, Wallin BG (1972) Manoeuvres affecting sympathetic outflow in human skin nerves. Acta Physiol Scand 84: 177-186

5. Bini G, Hagbarth K-E, Hynninen P, Wallin BG (1980) Regional similarities and differences in thermoregulatory vaso- and sudomotor tone. J Physiol 306: 553-565

6. Banauch D, Brümmer W, Ebeling W, Metz H, Rindfrey H, Lang H, Leybold K, Rick W (1975) Eine Glucose-Dehydrogenase für die Glucose-Bestimmung in Körperflüssigkeiten. Z Klin Chem Klin Biochem 13:101-107

7. Allenmark S, Hedman L, Söderberg A (1980) Microanalysis of catecholamines in human plasma by high-performance liquid chromatography with amperometric detection as compared with a radioenzymatic method. Microchem J 25: 567-575 
8. Fagius J, Blumberg H (1985) Sympathetic outflow to the hand in patients with Raynaud's phenomenon. Cardiovasc Res 19: 249-253

9. Fagius J, Sundlöf G (1986) The diving response in man: effects on sympathetic activity in muscle and skin nerve fascicles. J Physiol 377: 429-443

10. Fagius J, Wallin BG (1980) Sympathetic reflex latencies and conduction velocities in normal man. J Neurol Sci 47: 433-448

11. Bini G, Hagbarth K-E, Hynninen P, Wallin BG (1980) Thermoregulatory and rhythm-generating mechanisms governing the sudomotor and vasoconstrictor outflow in human cutaneous nerves. J Physiol 306: 537-552

12. Bini G, Hagbarth K-E, Wallin BG (1981) Cardiac rhythmicity of skin sympathetic activity recorded from peripheral nerves in man. J Aut Nerv Syst 4: 17-24

13. Gellhorn E (1938) Effects of hypoglycemia and anoxia on the central nervous system. Arch Neurol Psychiatry 40: 125-146

14. Gale EAM, Bennett T, MacDonald IA, Holst JJ, Matthews JA (1983) The physiological effects of insulin-induced hypoglycaemia in man: responses at differing levels of blood glucose. Clin Sci 65: $263-271$

15. Gale EAM, Bennett T, Hilary Green J, MacDonald IA (1981) Hypoglycaemia, hypothermia and shivering in man. Clin Sci 61: 463-469

16. MacDonald IA, Bennett T, Gale EAM, Hilary Green J, Walford S (1982) The effect of propranolol or metoprolol on thermoregulation during insulin-induced hypoglycaemia in man. Clin Sci 63: 301-310

17. Middleton WG, French EB (1974) Studies of the peripheral vasodilator response to acute insulin-induced hypoglycaemia in man. Clin Sci Mol Med 47: 461-470

18. Abramson DI, Schkloven N, Margolis MN, Mirsky IA (1939) Influence of massive doses of insulin on peripheral blood flow in man. Am J Physiol 128: 124-132
19. Alpert JS, Coffman JD (1969) Effect of intravenous epinephrine on skeletal muscle, skin, and subcutaneous blood flow. Am J Physiol 216: 156-160

20. Allwood MJ, Ginsburg J, Paton A (1957) The effect of insulin hypoglycaemia on blood flow in intact and sympathectomized extremities in man. J Physiol 139:97-107

21. Ginsburg J, Paton A (1956) Effects of insulin after adrenalectomy. Lancet 2: 491-494

22. Hilsted J, Bonde-Petersen F, Norgaard M-B, Greniman M, Christensen NJ, Parving H-H, Suzuki M (1984) Haemodynamic changes in insulin-induced hypoglycaemia in normal man. Diabetologia $26: 328-332$

23. Altorfer RM, Ziegler WH, Froesch ER (1981) Insulin hypoglycaemia in normal and adrenalectomized subjects: comparison of metabolic parameters and endocrine counter regulation. Acta Endocrinol 98: 413-419

24. Shah SD, Tse TF, Clutter WE, Cryer PE (1984) The human sympathochromaffin system. Am J Physiol 247: E380-E384

25. Clarke BF, Ewing DJ, Campbell IW (1979) Diabetic autonomic neuropathy. Diabetologia 17: 195-212

26. Fagius J (1982) Microneurographic findings in diabetic polyneuropathy with special reference to sympathetic nerve activity. Diabetologia 23: 415-420

27. Santiago JV, White NH, Skor DA, Levandoski LA, Bier DM, Cryer PE (1984) Defective glucose counterregulation limits intensive therapy of diabetes mellitus. Am J Physiol 247: E215-E220

Received: 3 June 1986; and in revised form: 7 October 1986

Dr. Christian Berne

Department of Internal Medicine

Uppsala University

Akademiska Sjukhuset

S-75185 Uppsala

Sweden 